\title{
q-Shock soliton evolution
}

\author{
Oktay K. Pashaev*, Sengul Nalci \\ Department of Mathematics, Izmir Institute of Technology, Urla-Izmir 35430, Turkey
}

\section{A R T I C L E I N F O}

\section{Article history:}

Received 13 October 2010

Accepted 20 June 2012

Available online 9 August 2012

\begin{abstract}
A B S T R A C T
By generating function based on Jackson's q-exponential function and the standard exponential function, we introduce a new q-analogue of Hermite and Kampe-de Feriet polynomials. In contrast to q-Hermite polynomials with triple recurrence relations similar to [1], our polynomials satisfy multiple term recurrence relations, which are derived by the q-logarithmic function. It allows us to introduce the q-Heat equation with standard time evolution and the q-deformed space derivative. We find solution of this equation in terms of q-Kampe-de Feriet polynomials with arbitrary number of moving zeros, and solved the initial value problem in operator form. By q-analog of the Cole-Hopf transformation we obtain a new q-deformed Burgers type nonlinear equation with cubic nonlinearity. Regular everywhere, single and multiple q-shock soliton solutions and their time evolution are studied. A novel, self-similarity property of the q-shock solitons is found. Their evolution shows regular character free of any singularities. The results are extended to the linear time dependent q-Schrödinger equation and its nonlinear q-Madelung fluid type representation.
\end{abstract}

(c) 2012 Elsevier Ltd. All rights reserved.

\section{Introduction}

It is well known that Burgers' equation in one dimension can be reduced via the Cole-Hopf transformation to the linear heat equation. It allows one to solve the initial value problem for the Burgers equation and get exact solutions in the form of shock solitons and their scattering. Recently, a new q-Burgers-type nonlinear heat equation with cubic nonlinearity and its linearization by the q-Cole-Hopf transformation in terms of q-Heat equation was introduced in [1]. Exact solutions of this equation in the form of q-shock solitons were derived. It was found that due to zeros of the q-exponential function, time evolution of these shock solitons develops singularity in a finite time. The origin of this singularity is related with q-deformation of both, the time and the space derivatives. Thus, one expects that for the q-Heat equation with deformed space derivative only, the time evolution could have the regular character. In the present paper we introduce the differen-

\footnotetext{
* Corresponding author.

E-mail address: oktaypashaev@iyte.edu.tr (O.K. Pashaev).
}

tial-q-difference Burgers type equation with cubic nonlinearity, which includes the standard time derivative and the q-deformed space derivative. By using the q-Cole-Hopf transformation, this nonlinear equation is linearized in terms of the q-Heat equation with the q-difference space derivative. By generating function based on Jackson's q-exponential function and the standard exponential function we introduce a new q-analog of Hermite and Kampede Feriet polynomials, representing moving poles solution for this q-Burgers equation. Then, we derive the operator solution of the initial value problem (IVP) for the q-Burgers equation in terms of the IVP for the q-Heat equation. We construct several particular solutions in the form of regular everywhere q-shock solitons. It turns out that the static q-shock soliton solution of our equation shows remarkable self-similarity property in space coordinate $x$, similar to [1]. However, in contrast to [1], time evolution of our q-shock solitons is regular for any finite time. By extending our results to the complex domain we introduce the time dependent Schrödinger equation with q-deformed dispersion and the complex wave function. As a solution of this equation we get the set of complex q-Kampe-de Feriet 
polynomials. By the complex q-Cole-Hopf transformation we obtain the complex q-Burgers-Madelung equation as a coupled two fluid system with complex velocity function.

\section{2. q-Hermite polynomials}

We define a q-analog of Hermite polynomials by the generating function as a product of Jackson's q-exponential function [2] and the standard exponential function

$e^{-t^{2}} e_{q}\left([2]_{q} t x\right)=\sum_{N=0}^{\infty} H_{N}(x ; q) \frac{t^{N}}{[N]_{q} !}$,

where Jackson's q-exponential function is defined by

$e_{q}(x)=\sum_{n=0}^{\infty} \frac{x^{n}}{[n]_{q} !}$,

$[n]_{q} !=[1]_{q}[2]_{q} \cdots[n]_{q}$ and q-number $[3]$,

$[n]_{q}=\frac{q^{n}-1}{q-1}$.

From the defining identity (1) for the q-Hermite polynomials it is not difficult to derive an explicit sum formula

$H_{N}(x ; q)=\sum_{k=0}^{[N / 2]} \frac{(-1)^{k}\left([2]_{q} x\right)^{N-2 k}[N]_{q} !}{k ![N-2 k]_{q} !}$.

This explicit sum makes it transparent in which way our polynomials $H_{N}(x ; q)$, q-extend the $H_{N}(x)$ and how they are different from the known ones in literature. Moreover, the generating function (1) and polynomials (2) are distinct also from the ones derived in our paper [1]. By q-differentiating the generating function (1) with respect to $x$ we derive the two-terms recurrence relation

$D_{x} H_{N}(x ; q)=[2]_{q}[N]_{q} H_{N-1}(x ; q)$,

where the q-derivative is defined as [3]

$D_{\chi} f(x)=\frac{f(q x)-f(x)}{(q-1) x}$

While by standard differentiating of (1) with respect to $t$ and using the next evident equality

$t \frac{d}{d t} e_{q}\left([2]_{q} x t\right)=x \frac{d}{d x} e_{q}\left([2]_{q} x t\right)=\sum_{n=0}^{\infty} n \frac{\left([2]_{q} x t\right)^{n}}{[n]_{q} !}$

we obtain another two-term recurrence relation

$\left(x \frac{d}{d x}-N\right) H_{N}(x ; q)=2[N]_{q}[N-1]_{q} H_{N-2}(x ; q)$.

Now by taking standard derivative of (1) with respect to $t$ and using definition of the q-logarithmic function [9]

$\operatorname{Ln}_{q}(1+z)=\sum_{N=1}^{\infty} \frac{(-1)^{N-1} z^{N}}{[N]}$,

where $q>1,0<|z|<q$ and the property

$\frac{d}{d z} \operatorname{Ln} e_{q}\left(\frac{\alpha z}{1-q}\right)=\frac{\operatorname{Ln}_{q}(1-\alpha z)}{(q-1) z}$

we derive the $\mathrm{N}$-term recurrence relation formula

$$
\begin{aligned}
H_{N+1}(x ; q)= & \frac{[N+1]_{q}}{N+1}\left\{[2]_{q} x H_{N}(x ; q)-2[N]_{q} H_{N-1}(x ; q)\right. \\
& -(q-1)[2]_{q}[N]_{q} x^{2} H_{N-1}(x ; q) \\
& \left.+[2]_{q}[N]_{q} ! \sum_{k=0}^{N-2} \frac{\left(1-q^{2}\right)^{N-k} x^{N-k+1} H_{k}(x ; q)}{[k]_{q} ![N-k+1]_{q}}\right\}
\end{aligned}
$$

or

$$
\begin{aligned}
H_{N+1} & =\frac{[N+1] !}{N+1}\left(-2 \frac{H_{N-1}}{[N-1] !}+\sum_{k=0}^{N} \frac{H_{k}(1-q)^{N-k}([2] x)^{N+1-k}}{[k] ![N+1-k]}\right) \\
& =\frac{[N+1]}{N+1}\left(-2[N] H_{N-1}+[N] ! \sum_{k=0}^{N} \frac{H_{k}(1-q)^{N-k}([2] x)^{N+1-k}}{[k] ![N+1-k]}\right),
\end{aligned}
$$

where $N=1,2, \ldots$. Here we emphasis that this $\mathrm{N}$-term recurrence relations can not be reduced to the typical three-term recurrence relations for Hermite and q-Hermite polynomials. This is why starting from $N=4$, our q-Hermite polynomials become different.

When $q \rightarrow 1$ this multiple term recurrence relation for q-Hermite polynomials reduces to the three-term recurrence relation for the standard Hermite polynomials

$H_{N+1}(x)=2 x H_{N}(x)-2 N H_{N-1}(x)$.

Substituting (3) into N-term recurrence relation formula we get the operator representation

$$
\begin{aligned}
H_{N+1}(x ; q)= & \frac{[N+1]_{q}}{N+1}\left([2]_{q} x-\left(\frac{2}{[2]_{q}}+(q-1) x^{2}\right) D_{x}\right. \\
& \left.+\sum_{l=2}^{N} \frac{\left(1-q^{2}\right)^{l} x^{l+1}}{[2]_{q}^{l-1}[l+1]_{q}} D_{x}^{l}\right) H_{N}(x ; q)
\end{aligned}
$$

By the recursion, starting from $n=0$ and $H_{0}(x ; q)=1$ we have the next operator representation for our q-Hermite polynomials

$$
\begin{aligned}
H_{N+1}(x ; q)= & \prod_{k=0}^{N} \frac{[k+1]_{q}}{k+1}\left([2]_{q} x-\left(\frac{2}{[2]_{q}}+(q-1) x^{2}\right) D_{x}\right. \\
& \left.+\sum_{k=2}^{N} \frac{\left(1-q^{2}\right)^{k} x^{k+1}}{[2]_{q}^{k-1}[k+1]_{q}} D_{x}^{k}\right) \cdot 1 .
\end{aligned}
$$

We notice that in contrast to known operator representations for Hermite and q-Hermite polynomials with only first order q-derivative [1], now we have the product, including higher order derivatives up to order $N$.

In the limit $q \rightarrow 1$ case this product formula is reduced to the known one

$H_{N}(x)=\left(2 x-\frac{d}{d x}\right)^{N} \cdot 1$

Here again we like to stress that the generating function and the form of our new q-Hermite polynomials are different from the known ones in the literature, [4-7], including our paper [1]. Moreover, instead of three-term recurrence relation we have multiple term recurrence relation, which shows that our q-Hermite polynomials are distinct from the known ones for orthogonal polynomial sets [8]. Comparison of above polynomials with ones obtained in [1], shows similarity up to $N=3$ only. Starting from $N=4$ they become different. 
The first few q-Hermite polynomials are

$$
\begin{aligned}
& H_{0}(x ; q)=1, \\
& H_{1}(x ; q)=[2]_{q} x, \\
& H_{2}(x ; q)=[2]_{q}^{2} x^{2}-[2]_{q}, \\
& H_{3}(x ; q)=[2]_{q}^{3} x^{3}-[3]_{q} ![2]_{q} x, \\
& H_{4}(x ; q)=[2]_{q}^{4} x^{4}-[4]_{q}[3]_{q}[2]_{q}^{2} x^{2}+\frac{1}{2}[4]_{q} !, \\
& H_{5}(x ; q)=[2]_{q}^{5} x^{5}-[5]_{q}[4]_{q}[2]_{q}^{3} x^{3}+\frac{1}{2}[5]_{q} ![2]_{q} x .
\end{aligned}
$$

\section{1. q-Difference equation}

By applying $D_{x}$ to both sides of (3) and using recurrence formula (5) we get mixed q-difference-differential equation for our q-Hermite polynomials

$2 D_{x}^{2} H_{N}(x ; q)-[2]_{q}^{2} x \frac{d}{d x} H_{N}(x ; q)+[2]_{q}^{2} N H_{N}(x ; q)=0$.

This form is different from [1] and in $q \rightarrow 1$ limit it reduces to the second order linear differential equation for the standard Hermite polynomials

$\frac{d^{2}}{d x^{2}} H_{N}(x)-2 x \frac{d}{d x} H_{N}(x)+2 N H_{N}(x)=0$.

\section{3. q-Kampe-de Feriet polynomials}

We define the q-Kampe-de Feriet polynomials as

$H_{N}(x, v t ; q)=(-v t)^{\frac{N}{2}} H_{N}\left(\frac{x}{[2]_{q} \sqrt{-v t}} ; q\right)$,

so that from the $\mathrm{N}$-term recurrence relation for q-Hermite polynomials we obtain $\mathrm{N}$-term recurrence relation formula for q-Kampe-de Feriet polynomials

$$
\begin{aligned}
H_{N+1}(x, v t ; q)= & \frac{[N+1]_{q}}{N+1}\left[x H_{N}(x, v t ; q)+2 v t[N]_{q} H_{N-1}(x, v t ; q)\right. \\
& -\frac{1}{[2]_{q}}(q-1)[N]_{q} x^{2} H_{N-1}(x, v t ; q) \\
& \left.+[N]_{q} ! \sum_{k=0}^{N-2} \frac{\left(1-q^{2}\right)^{N-k} x^{N-k+1} H_{k}(x, v t ; q)}{[k]_{q} ![N-k+1]_{q}[2]_{q}^{N-k}}\right]
\end{aligned}
$$

This can also be written in the operator form as

$$
\begin{aligned}
H_{N+1}(x, v t ; q)= & \frac{[N+1]_{q}}{N+1}\left[x+\left(2 v t+\frac{1-q}{[2]_{q}} x^{2}\right) D_{x}\right. \\
& \left.+\sum_{l=2}^{N} \frac{\left(1-q^{2}\right)^{l} x^{l+1}}{[2]_{q}^{l}[l]_{q}} D_{x}^{l}\right] H_{N}(x, v t ; q)
\end{aligned}
$$

By the recursion, starting from $n=0$ and $H_{0}(x, v t ; q)=1$ we have the next operator representation for the q-Kampe-de Feriet polynomials

$$
\begin{aligned}
H_{N+1}(x, v t ; q)= & \prod_{k=0}^{N} \frac{[k+1]_{q}}{k+1}\left[x+\left(2 v t+\frac{1-q}{[2]_{q}} x^{2}\right) D_{x}\right. \\
& \left.+\sum_{k=2}^{N} \frac{\left(1-q^{2}\right)^{k} x^{k+1}}{[2]_{q}^{k}[k]_{q}} D_{x}^{k}\right] \cdot 1
\end{aligned}
$$

In $q \rightarrow 1$ case it gives the Galilean bust form

$H_{N}(x, v t)=\left(x+2 v t \frac{d}{d x}\right)^{N} \cdot 1$.

For the first few q-Kampe-de Feriet polynomials we get

$H_{0}(x, v t ; q)=1$,

$H_{1}(x, v t ; q)=x$,

$H_{2}(x, v t ; q)=x^{2}+[2]_{q} v t$,

$H_{3}(x, v t ; q)=x^{3}+[2]_{q}[3]_{q} v t x$,

$H_{4}(x, v t ; q)=x^{4}+[3]_{q}[4]_{q} v t x^{2}+\frac{[4]_{q} !}{2} v^{2} t^{2}$,

$H_{5}(x, v t ; q)=x^{5}+[4]_{q}[5]_{q} v t x^{3}+\frac{[5]_{q} !}{2} v^{2} t^{2} x$.

When $q \rightarrow 1$ these polynomials reduce to the standard Kampe-de Feriet polynomials. As we commented in previous section, our new q-Kampe-de Feriet polynomials and corresponding representations are different from known ones, including our paper [1].

\section{4. q-Heat equation}

Now we introduce the q-Heat equation

$\left(\partial_{t}-v D_{x}^{2}\right) \phi(x, t)=0$

with partial q-derivative with respect to $x$ and with partial standard derivative in time $t$. In contrast with this equation, in our paper [1] we worked with different q-Heat equation, where both, the time and the space derivatives are q-derivatives.

By separation of variables one can easily see that

$\phi_{k}(x, t)=e^{v k^{2} t} e_{q}(k x)$

is a particular plane wave solution of (13). By expanding this in terms of parameter $k$

$\phi_{k}(x, t)=e^{v k^{2} t} e_{q}(k x)=\sum_{N=0}^{\infty} H_{N}(x, v t ; q) \frac{k^{N}}{[N]_{q} !}$

we get the set of q-Kampe-de Feriet polynomial solutions for the q-Heat Eq. (13). From the defining identity (14) it is not difficult to derive an explicit sum formula for these polynomials

$H_{N}(x, v t ; q)=\sum_{k=0}^{[N / 2]} \frac{(v t)^{k} x^{N-2 k}[N]_{q} !}{k ![N-2 k]_{q} !}$.

\subsection{Operator representation}

\section{Proposition 1}

$e^{-\frac{1}{[2]_{q}^{2}} D_{x}^{2}} e_{q}\left([2]_{q} x t\right)=e^{-t^{2}} e_{q}\left([2]_{q} x t\right)$.

Proof 1. By q-differentiating the q-exponential function with respect to $x$ 
$D_{x}^{n} e_{q}\left([2]_{q} x t\right)=([2] t)^{n} e_{q}\left([2]_{q} x t\right)$,

and combining then to the sum

$\sum_{n=0}^{\infty} \frac{a^{n}}{n !} D_{x}^{2 n} e_{q}\left([2]_{q} x t\right)=\sum_{n=0}^{\infty} \frac{\left([2]_{q} t\right)^{2 n} a^{n}}{n !} e_{q}\left([2]_{q} x t\right)$,

we have relation

$e^{a D_{x}^{2}} e_{q}\left([2]_{q} x t\right)=e^{a\left([2]_{q} t\right)^{2}} e_{q}\left([2]_{q} x t\right)$.

By choosing $a=-1 /[2]_{q}^{2}$ we get the result (16).

\section{Proposition 2}

$H_{N}(x ; q)=[2]_{q}^{N} e^{-\frac{1}{[2]_{q}^{2}} D_{x}^{2}} x^{N}$.

Proof 2. The right hand side of (16) is the generating function for the q-Hermite polynomials (1). Hence, equating the coefficients of $t^{n}$ on both sides gives the result.

Corollary 1. If function $f(x)$ is expandable to the formal power series $f(x)=\sum_{N=0}^{\infty} a_{N} x^{N}$ then we have the next q-Hermite series expansion

$e^{-\frac{1}{[2]_{q}^{2}} D_{x}^{2}} f(x)=\sum_{N=0}^{\infty} a_{N} \frac{H_{N}(x ; q)}{[2]_{q}^{N}}$

\section{Evolution operator}

Following similar calculations as in Proposition 1 we have the next relation

$e^{v t D_{x}^{2}} e_{q}(k x)=e^{v t k^{2}} e_{q}(k x)$.

The right hand side of this expression is the plane wave type solution of the q-Heat Eq. (13). Expanding both sides in power series in $k$ and equating the coefficients of $k^{N}$ on both sides, we get q-Kampe de Feriet polynomial solutions of this equation

$H_{N}(x, v t ; q)=e^{v t D_{x}^{2}} \chi^{N}$

as solution of the I.V.P. with $\phi(x, 0)=x^{N}$.

Consider an arbitrary, expandable to the power series function $f(x)=\sum_{n=0}^{\infty} a_{n} x^{n}$, then the formal series

$$
\begin{aligned}
f(x, t)=e^{v t D_{x}^{2}} f(x) & =\sum_{n=0}^{\infty} a_{n} e^{v t D_{x}^{2}} x^{n} \\
& =\sum_{n=0}^{\infty} a_{n} H_{N}(x, v t ; q),
\end{aligned}
$$

represents a time dependent solution of the q-Heat Eq. (13) The domain of convergency for this series is determined by asymptotic properties of our q-Kampe-de Feriet polynomials for $n \rightarrow \infty$ and requires additional study.

According to this we have the evolution operator for the q-Heat equation as

$U(t)=e^{v t D_{x}^{2}}$.
It allows us to solve the initial value problem

$$
\begin{aligned}
& \left(\frac{\partial}{\partial t}-v D_{x}^{2}\right) \phi(x, t)=0, \\
& \phi(x, 0)=f(x),
\end{aligned}
$$

in the form

$\phi(x, t)=e^{v t D_{x}^{2}} \phi(x, 0)=e^{v t D_{x}^{2}} f(x)$,

where we imply the base $q>1$ so that $e_{q}(x)$ is an entire function.

\section{6. q-Burgers' type equation}

By using the q-Cole-Hopf transformation [1]

$u(x, t)=-2 v \frac{D_{x} \phi(x, t)}{\phi(x, t)}$

where $\phi(x, t)$ is a solution of the q-Heat Eq. (13), we find then that $u(x, t)$ satisfies the q-Burgers' type evolution equation with cubic nonlinearity

$$
\begin{aligned}
\frac{\partial}{\partial t} u(x, t)-v D_{x}^{2} u(x, t)= & \frac{1}{2}\left[\left(1-M_{q}^{x}\right) u(x, t) D_{x} u(x, t)\right] \\
& -\frac{1}{2}\left[D_{x}(u(q x, t) u(x, t))\right]+\frac{1}{4 v}\left[u\left(q^{2} x, t\right)\right. \\
& -u(x, q t)] u(q x, t) u(x, t),
\end{aligned}
$$

where $M_{q}^{x}$ is the dilatation operator $M_{q}^{x} f(x)=f(q x)$.

In contrast to q-Burgers equation from [1], including the space and the time q-derivatives, now we have nonlinear q-space derivative equation with standard time evolution.

When $q \rightarrow 1$ this equation reduces to Burgers' Equation

$u_{t}+u u_{x}=v u_{x x}$

\subsection{I.V.P. for q-Burgers' type equation}

Substituting the operator solution (29) and (30) we find operator solution for the q-Burgers type equation in the form

$u(x, t)=-2 v \frac{e^{v t D_{x}^{2}} D_{x} f(x)}{e^{v t D_{x}^{2}} f(x)}$.

This solution corresponds to the initial function

$u(x, 0)=-2 v \frac{D_{\chi} f(x)}{f(x)}$

Thus, for arbitrary initial value $u(x, 0)=F(x)$ for the q-Burgers equation we need to solve the initial value problem for the q-Heat Eq. (13) with initial function $f(x)$ satisfying the first order q-difference equation

$$
\left(D_{x}+\frac{1}{2 v} F(x)\right) f(x)=0 .
$$

\section{7. q-Shock soliton solution}

As a particular solution of the q-Heat equation we choose first 
$\phi(x, t)=e^{k^{2} t} e_{q}(k x)$,

then we find solution of the q-Burgers equation as a constant

$u(x, t)=-2 v k$.

We notice that for this solution of the q-Heat equation, we have an infinite set of zeros, and the space position of zeros is fixed during time evolution at points $x_{n}=-q^{n+1} /(q-1) k$, $n=0,1, \ldots$.

For the linear superposition

$\phi(x, t)=e^{k_{1}^{2} t} e_{q}\left(k_{1} x\right)+e^{k_{2}^{2} t} e_{q}\left(k_{2} x\right)$,

we have the q-shock soliton solution

$u(x, t)=-2 v \frac{k_{1} e^{k_{1}^{2} t} e_{q}\left(k_{1} x\right)+k_{2} e^{k_{2}^{2} t} e_{q}\left(k_{2} x\right)}{e^{k_{1}^{2} t} e_{q}\left(k_{1} x\right)+e^{k_{2}^{2} t} e_{q}\left(k_{2} x\right)}$.

This expression is the q-analog of the Burgers shock soliton and for $q \rightarrow 1$ it reduces to the last one. However, in contrast to the standard Burgers case, due to zeroes of the qexponential function, this expression admits singularities coming from $x$ and determined in terms of parameters $k_{1}$ and $k_{2}$.To have regular solution we can follow the similar approach as discussed in [1]. Then for $k_{2}=-k_{1}$ we have the stationary shock soliton

$$
\begin{aligned}
u(x, t) & =-2 v k_{1} \frac{e_{q}\left(k_{1} x\right)-e_{q}\left(-k_{1} x\right)}{e_{q}\left(k_{1} x\right)+e_{q}\left(-k_{1} x\right)} \\
& =-2 v k_{1} \frac{\sinh _{q}\left(k_{1} x\right)}{\cosh _{q}\left(k_{1} x\right)} \equiv-2 v k_{1} \tanh _{q}\left(k_{1} x\right) .
\end{aligned}
$$

Since this shock soliton is time independent, as we can expect it coincides with the stationary one obtained in [1]. The function has no singularities on the real axis $x$ and the q-shock soliton is regular everywhere. However time evolution of shock solitons in [1] produce singularity at a finite time. In contrast to this, here we like to show existence of a shock soliton, which is regular in $x$ and continues to be regular at any time. For this we consider solution of q-Heat Eq. (13) in the form

$\phi(x, t)=10+e^{k_{1}^{2} t} e_{q}\left(k_{1} x\right)+e^{k_{2}^{2} t} e_{q}\left(k_{2} x\right)$,

then for $k_{1}=1$ and $k_{2}=-1$ we get the q-shock soliton

$u(x, t)=-2 v \frac{e_{q}(x)-e_{q}(-x)}{10 e^{-t}+e_{q}(x)+e_{q}(-x)}$.

Due to absence of zeros for $e^{-t}$ and the above discussion, it is easy to see that our q-shock is regular for any time $t$. The solution describes creation of the shock soliton, so that at $t \rightarrow-\infty, u(x, t) \rightarrow 0$, and for $t \rightarrow \infty, u(x, t) \rightarrow-2 v \tanh _{q} x$. In Figs. 1-3 we plot the regular q-shock soliton for $k_{1}=1$ and $k_{2}=-1$ at different times $t=-2,0,5$ with base $q=10$.

In Figs. 4-6 we plot the regular q-shock soliton evolution for $k_{1}=1$ and $k_{2}=-1$ at different ranges of $x$ and with $q=10$. It is remarkable fact that the structure of our qshock soliton shows self-similar property in the space coordinate $x$. Indeed at the range of parameter $-50<x<50$, Fig. 4 , and $-5000<x<5000$, Fig. 6 , the shape of shocks looks almost the same.

For the set of arbitrary numbers $k_{1}, \ldots, k_{N}$

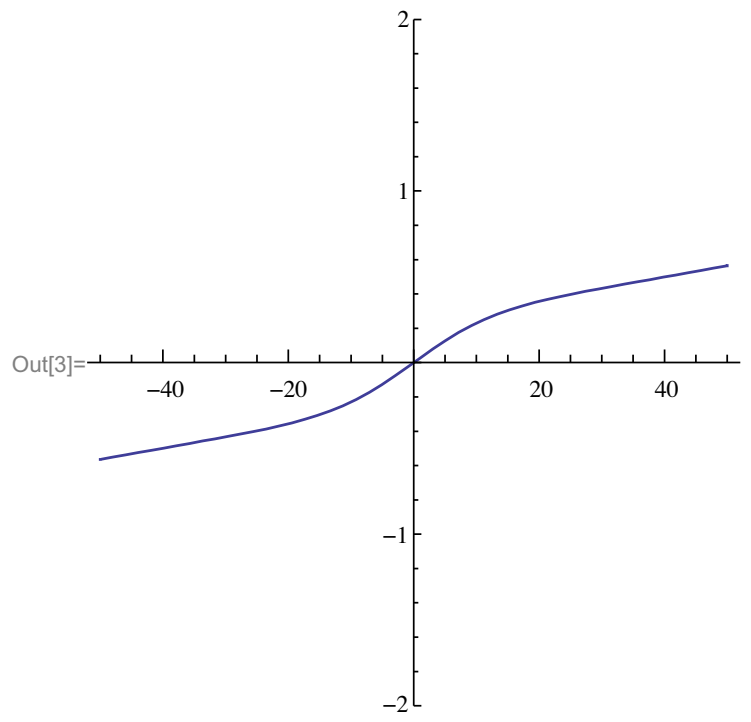

Fig. 1. q-shock evolution for $v=1, k_{1}=1, k_{2}=-1, t=-2$ at range $(-50,50)$.

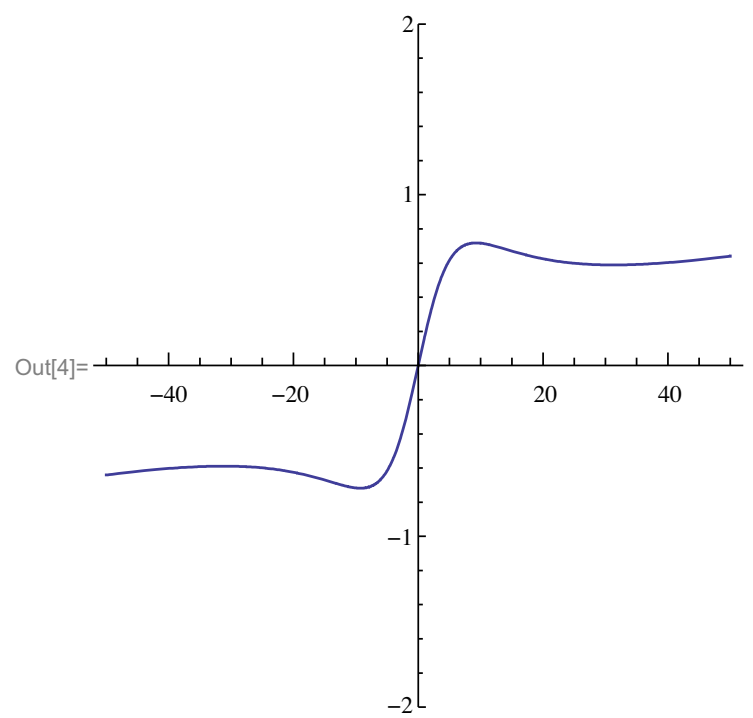

Fig. 2. q-shock evolution for $v=1, k_{1}=1, k_{2}=-1, t=0$ at range $(-50,50)$.

$\phi(x, t)=\sum_{n=1}^{N} e^{k_{n}^{2} t} e_{q}\left(k_{n} x\right)$

we have multi-shock solution in the form

$u(x, t)=-2 v \frac{\sum_{n=1}^{N} k_{n} e^{k_{n}^{2} t} e_{q}\left(k_{n} x\right)}{\sum_{n=1}^{N} e^{k_{n}^{2} t} e_{q}\left(k_{n} x\right)}$.

In general this solution admits several singularities. To have a regular multi-shock solution we take the even number of terms $N=2 k$ with opposite wave numbers. When $N=4$ and $k_{1}=1, k_{2}=-1, k_{3}=2, k_{4}=-2$ we have q-multishock soliton solution, 


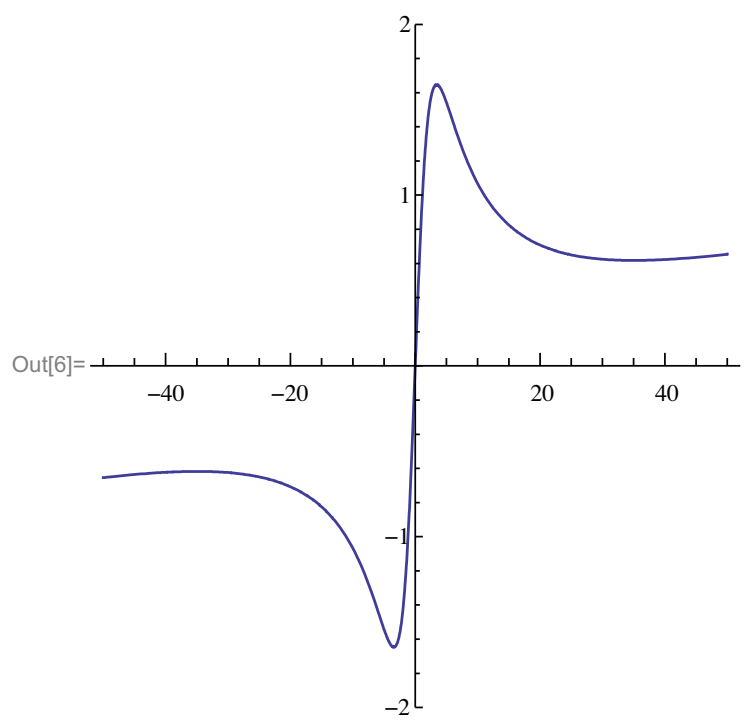

Fig. 3. q-shock evolution for $v=1, k_{1}=1, k_{2}=-1, t=5$ at range $(-50,50)$.

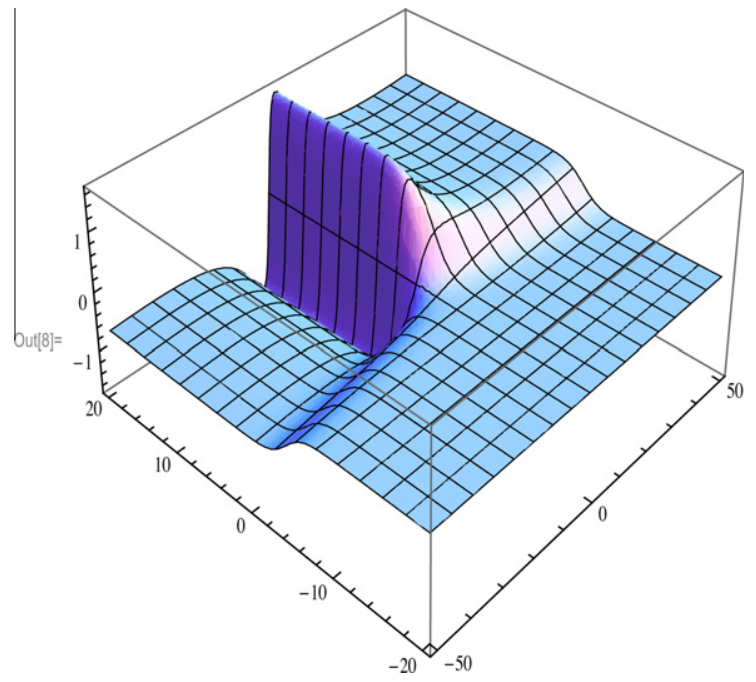

Fig. 4. q-shock evolution for $k_{1}=1, k_{2}=-1$, at range $(-50,50)$.

$u(x, t)=-2 v \frac{\sinh _{q}(x)+2 e^{3 t} \sinh _{q}(2 x)}{\cosh _{q}(x)+e^{3 t} \cosh _{q}(2 x)}$.

In Figs. 7-9 we plot $N=4$ case with values of the wave numbers $k_{1}=1, k_{2}=-1, k_{3}=2, k_{4}=-2$ at $t=-10,0,7$ and with $q=10$. This multi-shock soliton is regular everywhere in $x$ for arbitrary time $t$. Similar to the one q-shock case, this result takes place due to absence of zeros for the standard exponential function $e^{k^{2} t}$.

In Figs. 10-12 we plot this regular multi q-shock soliton evolution at different ranges of $x$. It is remarkable that the structure of this regular multi q-shock soliton shows also the self-similar property in the space coordinate $x$. Indeed at the range of parameter $-50<x<50$, Fig. 10, and $-5000<x<5000$, Fig. 12, structure of this multi q-shocks looks almost the same.

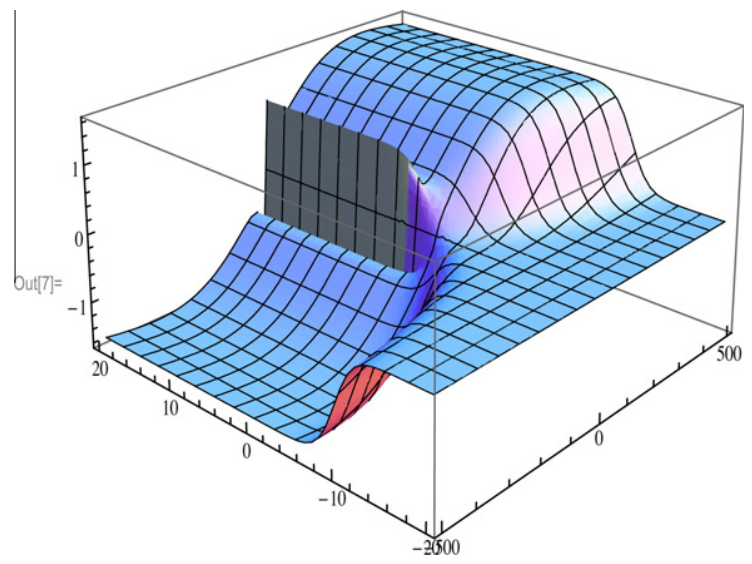

Fig. 5. q-shock evolution for $k_{1}=1, k_{2}=-1$, at range $(-500,500)$.

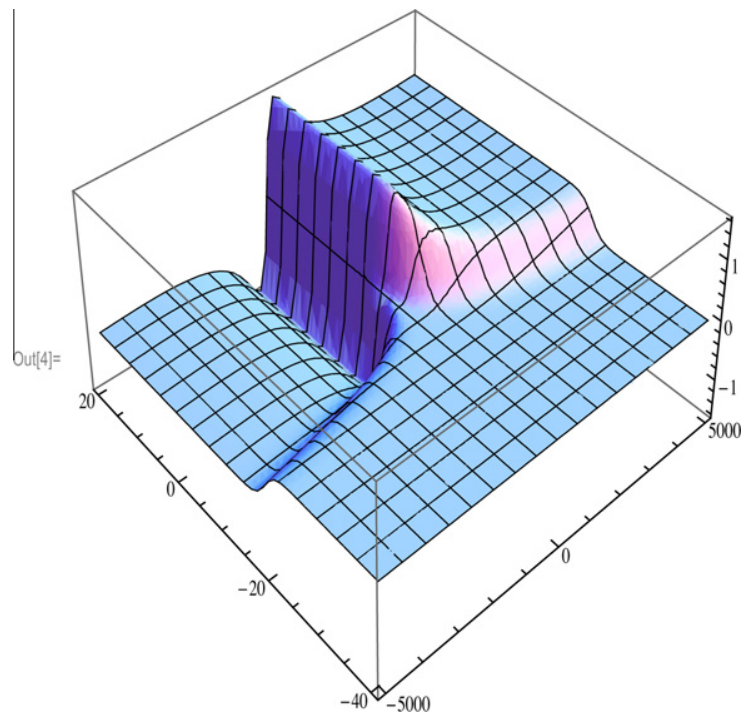

Fig. 6. q-shock evolution for $k_{1}=1, k_{2}=-1$, at range $(-5000,5000)$.

\section{The q-Schrödinger equation}

The above consideration can be extended to the time dependent Schödinger equation with q-deformed dispersion. We consider the standard time-dependent q-Schödinger equation

$\left(\frac{\partial}{\partial t}-\frac{i \hbar}{2 m} D_{x}^{2}\right) \psi(x, t)=0$

where $\psi(x, t)$ is a complex wave function.

One can easily see that

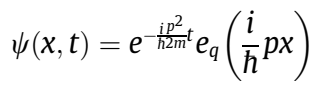

is the plane wave solution of (43). By expanding this in terms of momentum $p$ 


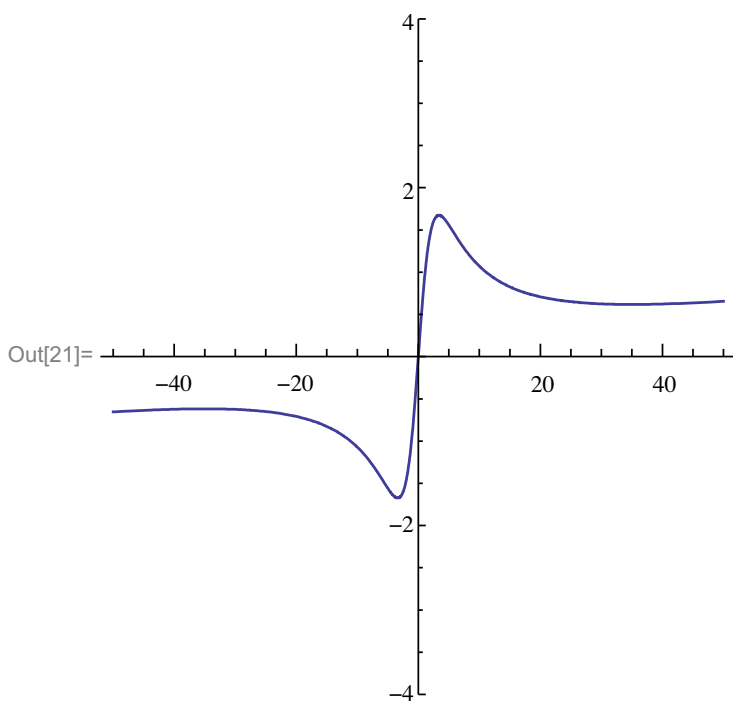

Fig. 7. q-multi shock evolution for $k_{1}=1, k_{2}=-1, k_{3}=2, k_{4}=-2, t=-10$ and at range $(-50,50)$.

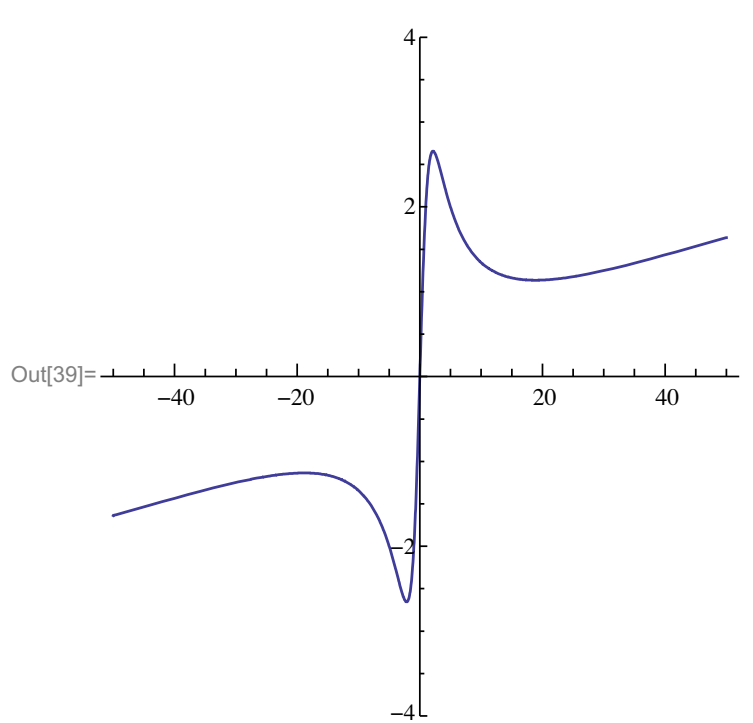

Fig. 8. q-multi shock evolution for $k_{1}=1, k_{2}=-1, k_{3}=2, k_{4}=-2, t=0$ and at range $(-50,50)$.

$\psi(x, t)=e^{-\frac{i p^{2}}{h 2 m} t} e_{q}\left(\frac{i}{h} p x\right)=\sum_{N=0}^{\infty}\left(\frac{i}{h}\right)^{N} \frac{p^{N}}{[N]_{q} !} H_{N}^{(s)}(x, i t ; q)$

we get the set of complex q-Kampe-de Feriet polynomial solutions of (43) as

$H_{N}^{(s)}(x, i t ; q)=\sum_{k=0}^{[N / 2]} \frac{\left(\frac{i h t}{2 m}\right)^{k}[N]_{q} ! x^{N-2 k}}{[N-2 k]_{q} k !}$.

We introduce complex version of the q-Cole-Hopf transformation, which plays role of the Madelung representation,

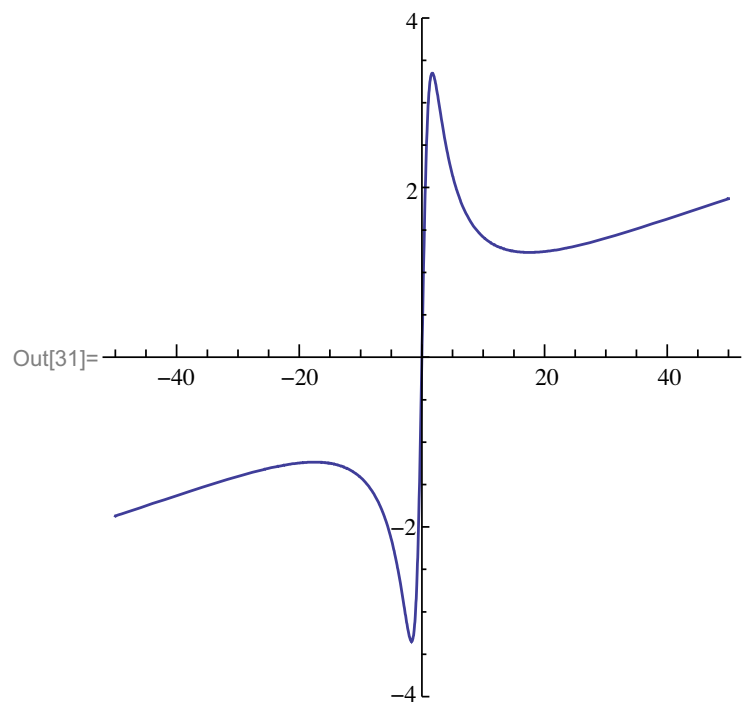

Fig. 9. q-multi shock evolution for $k_{1}=1, k_{2}=-1, k_{3}=2, k_{4}=-2, t=7$ and at range $(-50,50)$.

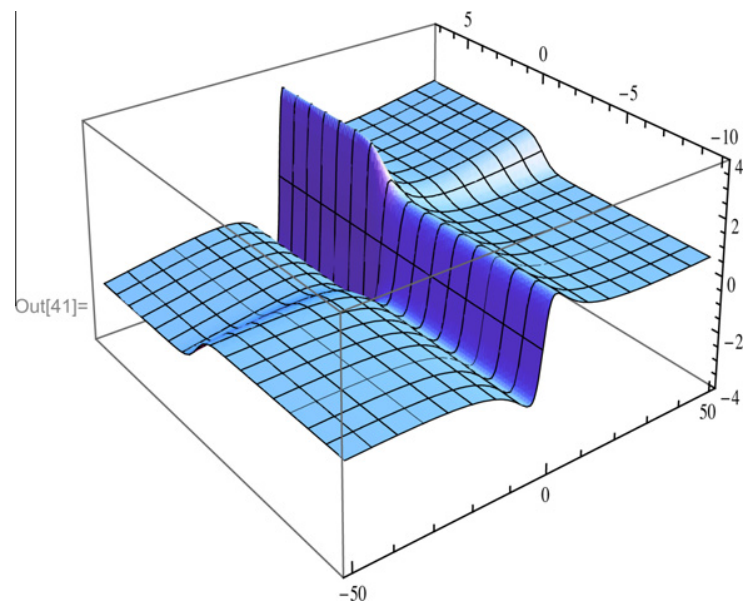

Fig. 10. q-multi shock evolution for $k_{1}=1, k_{2}=-1, k_{3}=2, k_{4}=-2$ at range $(-50,50)$.

$u(x, t)=-\frac{i \hbar}{m} \frac{D_{x} \psi(x, t)}{\psi(x, t)}$

Then, the complex velocity function $u(x, t)$ satisfies the complex q-Burgers-Madelung type equation

$$
\begin{aligned}
i \hbar \frac{\partial}{\partial t} u(x, t)+\frac{\hbar^{2}}{2 m} D_{x}^{2} u(x, t)= & \frac{i h}{2} u(x, t)\left[1-M_{q}^{x}\right] D_{x} u(x, t) \\
& -\frac{i h}{2}\left[D_{x}(u(q x, t) u(x, t))\right] \\
& +\frac{m}{2}\left[u\left(q^{2} x, t\right)-u(x, t)\right] u(q x, t) u(x, t) .
\end{aligned}
$$

If we separate $u=u_{1}+i u_{2}$ into real and imaginary parts, then we get two fluid model representation, where $u_{1}$ is the Madelung-London-Landau classical velocity, and $u_{2}$ is the "quantum velocity". 


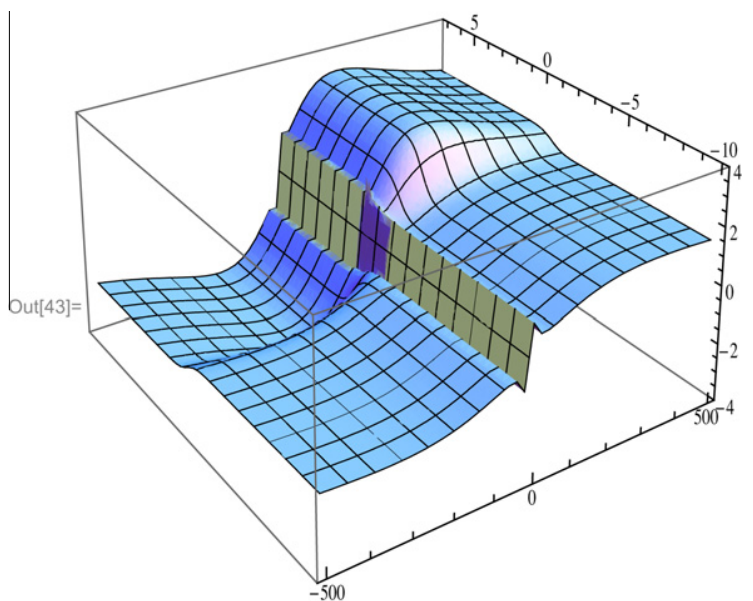

Fig. 11. q-multi shock evolution for $k_{1}=1, k_{2}=-1, k_{3}=2, k_{4}=-2$ at range $(-500,500)$.

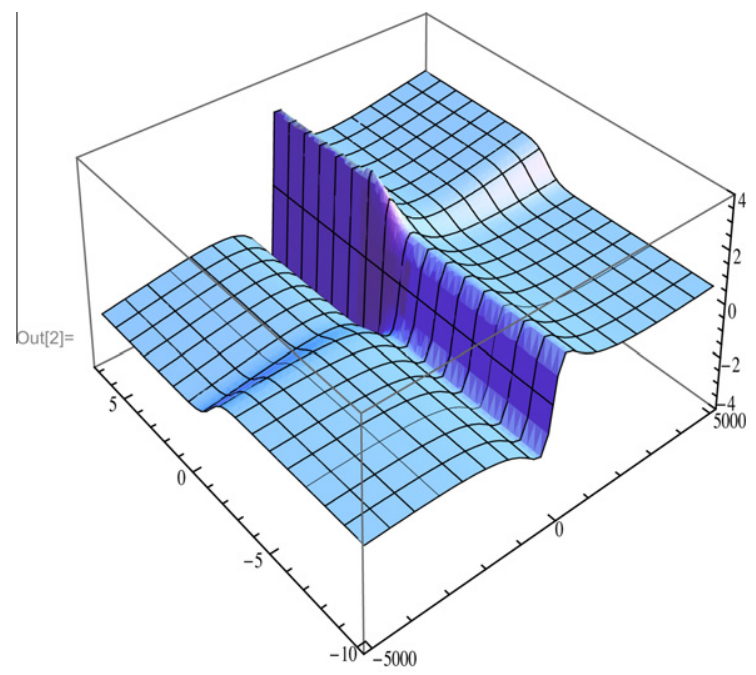

Fig. 12. q-multi shock evolution for $k_{1}=1, k_{2}=-1, k_{3}=2, k_{4}=-2$ at range $(-5000,5000)$.

For the real part we have

$-h \frac{\partial}{\partial t} u_{2}(x, t)+\frac{h^{2}}{2 m} D_{x}^{2} u_{1}(x, t)=\frac{m}{2}\left[\left(u_{1}\left(q^{2} x, t\right)-u_{1}(x, t)\right)\right.$

$$
\begin{aligned}
& \times\left(u_{1}(x, t) u_{1}(q x, t)-u_{2}(x, t) u_{2}(q x, t)\right) \\
& -\left(u_{2}\left(q^{2} x, t\right)-u_{2}(x, t)\right)\left(u_{1}(x, t) u_{2}(q x, t)\right. \\
& \left.\left.+u_{2}(x, t) u_{1}(q x, t)\right)\right] \\
& -\frac{h}{2}\left[u_{1}(x, t)\left[1-M_{q}^{x}\right] D_{x} u_{2}(x, t)\right. \\
& \left.+u_{2}(x, t)\left[1-M_{q}^{x}\right] D_{x} u_{1}(x, t)\right] \\
& +\frac{h}{2} D_{x}\left[u_{2}(q x, t) u_{1}(x, t)\right. \\
& \left.+u_{1}(q x, t) u_{2}(x, t)\right],
\end{aligned}
$$

and for the imaginary part

$$
\begin{aligned}
h \frac{\partial}{\partial t} u_{1}(x, t)+\frac{h^{2}}{2 m} D_{x}^{2} u_{2}(x, t)= & \frac{m}{2}\left[\left(u_{1}\left(q^{2} x, t\right)\right.\right. \\
& \left.-u_{1}(x, t)\right)\left(u_{1}(x, t) u_{2}(q x, t)\right. \\
& \left.+u_{2}(x, t) u_{1}(q x, t)\right)+\left(u_{2}\left(q^{2} x, t\right)\right. \\
& \left.-u_{2}(x, t)\right)\left(u_{1}(x, t) u_{1}(q x, t)\right. \\
& \left.\left.-u_{2}(x, t) u_{2}(q x, t)\right)\right] \\
& +\frac{h}{2}\left[u_{1}(x, t)\left[1-M_{q}^{x}\right] D_{x} u_{1}(x, t)\right. \\
& \left.-u_{2}(x, t)\left[1-M_{q}^{x}\right] D_{x} u_{2}(x, t)\right] \\
& -\frac{h}{2} D_{x}\left[u_{1}(q x, t) u_{1}(x, t)\right. \\
& \left.-u_{2}(q x, t) u_{2}(x, t)\right] .
\end{aligned}
$$

When $q \rightarrow 1$, the real part reduces to the continuity equation

$-\left(u_{2}\right)_{t}+\frac{h}{2 m}\left(u_{1}\right)_{x x}=\left(u_{1} u_{2}\right)_{x}$,

and the imaginary part reduces to the Quantum HamiltonJacobi equation

$\left(u_{1}\right)_{t}+\frac{h}{2 m}\left(u_{2}\right)_{x x}=-\frac{1}{2}\left(u_{1}^{2}-u_{2}^{2}\right)_{x}$.

For $u_{1} \equiv v$ and $u_{2}=-\frac{h}{2 m}(\ln \rho)_{x}$ where $\rho=|\psi|^{2}$, the continuity equation is

$\rho_{t}+(\rho v)_{x}=0$

and the Euler equation with the quantum potential pressure term is

$$
v_{t}+v v_{x}=\left(\frac{h^{2}}{2 m^{2}} \frac{(\sqrt{\rho})_{x x}}{\sqrt{\rho}}\right)_{x} .
$$

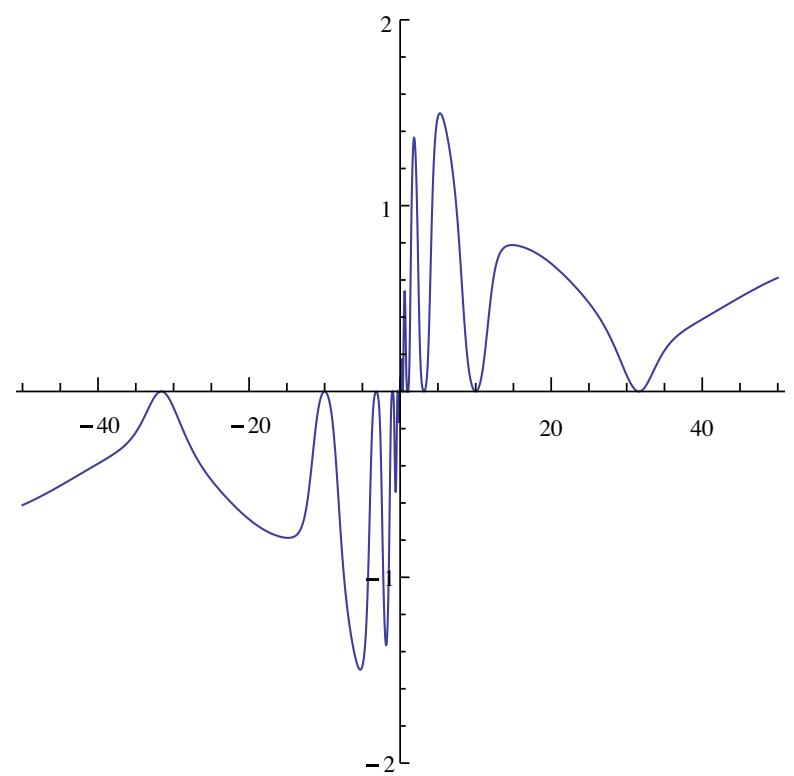

Fig. 13. q-modulated q-shock evolution for $k_{1}=-k_{2}=\sin \left(2 \pi \log _{10} x\right), t=5$ at range $(-50,50)$. 
Thus the two fluid system (44), (45) is the q-analogue of the coupled q-quantum Hamilton-Jacobi equation and the q-continuity equation.

Following similar procedure as in first part of this paper, we can construct particular solutions of our q-Schrödinger equation in the form of complex shock solitons. This question is under investigation now.

In conclusion we like to mention that all solutions presented above, can be extended to the case when arbitrary constants in space variables are the q-periodic functions of $x$. In this case our q-shock solitons possess an oscillating microstructure. As an illustration of this type of solution in Fig. 13 we show one q-shock soliton from Fig. 3, modulated by q-periodic function $k(x)=\sin \left(2 \pi \log _{10} x\right)$. This figure should be considered as q-modulated version of Fig. 3 .

Finally, recently we constructed q-version of the heat equation with the golden ratio basis and with Fibonacci derivatives [10]. The Burgers version of this equation and corresponding shock solitons are in progress.

\section{Acknowledgments}

This work was supported by TÜBITAK, Project No: $110 \mathrm{~T} 679$ and Izmir Institute of Technology. One of the authors (S. Nalci) was supported by TUBITAK Scholarship for Graduate Students.

\section{References}

[1] Nalci S, Pashaev OK. q-Analog of shock soliton solution. J Phys A Math Theor 2010;43:445205.

[2] Jackson FH. A basic sine and cosine with symbolic solutions of certain differential equations. Proc Edin Math Soc 1904;22:28-39.

[3] Kac V, Cheung P. Quantum calculus. New York: Springer; 2002.

[4] Exton H. q-Hypergeometric functions and applications. John Wiley and Sons; 1983.

[5] Rajkovic P, Marinkovic S. On Q-analogies of generalized Hermite's polynomials. Filomat 2001;15:277.

[6] Cigler J, Zeng J. Two curious q-Analogues of Hermite polynomials arXiv:0905.0228, 2009.

[7] J. Negro, The factorization method and hierarchies of q-oscillator Hamiltonians Centre de Recherches Mathematiques CRM, In: Proceedings and Lecture Notes, vol. 9, 1996, p. 239.

[8] Ismail M. Classical and quantum orthogonal polynomials in one variable. Cambridge University Press; 2005.

[9] Pashaev OK, Yilmaz O. Vortex images and q-elementary functions. J Phys A Math Theor 2008;41:135207.

[10] Pashaev OK, Nalci S. Golden quantum oscillator and Binet-Fibonacci calculus. J Phys A Math Theor 2012;45:015303. 